\title{
Practical use of distributed fibre optic sensors in research on FRCM composites
}

\author{
Filip Grzymski ${ }^{*}$, Tomasz Trapko , and Michał Musiał \\ Wrocław University of Science and Technology, Faculty of Civil Engineering, Pl. Grunwaldzki 11, \\ 50-377 Wroclaw, Poland
}

\begin{abstract}
This article describes research on FRCM (Fabric Reinforced Cementitious Matrix) composites, which unlike commonly used FRP (Fibre Reinforced Polymers) composites make use of a mineral matrix instead of epoxy resin, which allows to achieve much higher resistance to elevated temperatures. In the described studies, experimental measurement of deformations with the use of the DFOS (Distributed Fibre Optic Sensors) method was applied. This method allows for geometrically continuous measurement of deformations, which is its significant advantage compared to traditional electric resistance wire strain gauge, as it reduces the possibility of measuring deformations in a place where they are not representative. The tests were carried out using two reinforced concrete slab elements loaded to failure in the 4-point bending scheme. Fibre optic sensors were installed on an unstrengthened control element and on an element strengthened with FRCM composite. During the tests, deformations of the concrete under tension and the external surface of the FRCM reinforcing composite were determined. Measurements were carried out simultaneously in two manners: using the DFOS method, and strain gauges placed at the characteristic points of the element. The test results based on both methods were compared and analysed. The comparative analysis confirmed the usefulness and effectiveness of the DFOS method while measuring deformations in strengthening composites, and showed its significant advantages such as precise indication of the place of elements cracking as well as the possibility of conducting a global analysis of deformations.
\end{abstract}

\section{Introduction}

Effectiveness of strengthening composites work in reinforced concrete structures is most often assessed in destructive tests, in which the force carried by the element is determined. Composite deformation is of great importance in this type of research, since on its basis, it is possible to assess the utilization of the material which high-strength fibres are made of. This is particularly important in the case of composites in which the mechanism of destruction is not related to fibre rupture, but to their slipping or composite delamination.

FRCM composites (Fabric Reinforced Cementitious Matrix), unlike FRP composites (Fibre Reinforced Polymers), use a mineral matrix instead of an epoxy resin matrix.

\footnotetext{
* Corresponding author: filip.grzymski@pwr.edu.pl
} 
This kind of modification allows for a significant increase in material resistance to elevated temperature [1]. The change of matrix, from the epoxy to mineral one, also affects the work mechanism of the material, due to its different mechanical parameters. FRCM composites usually assume a two-line or three-line mechanism of work, in contrast to the one-line mechanism observed in FRP composites [2, 3]. An additional factor having a significant impact on the mechanism of FRCM composites destruction is the adhesion of the matrix to concrete, resulting from the preparation of the surface and shrinkage deformations during the bonding of the mineral mortar [4]. The research described in the literature shows that better utilization of mesh in the FRCM composite can be obtained by means of its appropriate anchorage [5, 6]. FRCM composites undergo local deformations at the cracking area of a reinforced concrete element, therefore it is advisable to monitor their entire surface while they are being tested.

One method to geometrically measure deformations is to employ Distributed Fibre Optic Sensors (DFOS). This method is part of the field of opto-electronics, and uses the physical phenomena such as total internal reflection of light or Rayleigh scattering [7] or Raman and Brillouin scattering [8]. Measurement with the use of DFOS consists in passing the light through the optical fibre installed on an element and measuring the distance of microdefects present in it with a reflectometer. On the basis of changes in their location as well as mathematical transformations, it is possible to determine the deformations of the element. Fibre optic sensors have found practical applications in structural health monitoring, and have great development potential [9], however, their use in the laboratory is also possible. Continuously distributed fibre optic sensors are particularly useful in determining the location of cracks and other defects of the structure, which is their most important advantage compared to other electronic sensors normally used $[10,11]$.

\section{Methods}

The research carried out was aimed at checking the usefulness of DFOS method in determining the deformation of FRCM composites. Two reinforced concrete slab elements were used for testing, one of which was strengthened with the PBO-FRCM composite.

\subsection{Experimental setup and materials}

Reinforced concrete slabs with a length of $2000 \mathrm{~mm}$, a width of $1000 \mathrm{~mm}$ and thickness of $150 \mathrm{~mm}$ were tested in the 4-point bending scheme. The distance between the supports of the element was $1700 \mathrm{~mm}$, and the loading forces were applied to the element in the spacing equal to $1 / 3$ of the length of the span.

The bottom reinforcement of the slabs was made of 8 bars with a diameter of $10 \mathrm{~mm}$, and 8 bars with a diameter of $8 \mathrm{~mm}$ were used as the upper reinforcement. Additional transverse reinforcement was made of bars with a diameter of $8 \mathrm{~mm}$ arranged at the top and bottom at a spacing of $200 \mathrm{~mm}$. All reinforcing bars were made of steel with a characteristic yield strength of $500 \mathrm{MPa}$. The concrete used for making the samples was marked by an average compressive strength of $38.8 \mathrm{MPa}$ and Young's modulus of $35.2 \mathrm{GPa}$. A PBO (p-Phenylene BenzobisOxazole) fibre mesh with a tensile strength of $5800 \mathrm{MPa}$ and Young's $270 \mathrm{GPa}$ modulus was used for the FRCM composite [12]. The composite mesh was anchored at the ends by winding it upon a GFRP (Glass Fibre Reinforced Polymer) bar, which was then placed in a specially prepared groove in the slab. 


\subsection{Measurements of deformations}

Deformations of elements were measured with the use of two methods: DFOS and strain gauges. The deformations were compared on the concrete under tension, and in the case of the strengthened element also on the outer surface of the PBO-FRCM composite. Optical fibre sensors on the concrete surface were laid in six rows in the longitudinal direction of the element and in two rows in the transverse direction. The same distribution of fibre optic sensors was used on the surface of the composite. Paper strain gauges with a $50 \mathrm{~mm}$ measuring base were glued in the form of three cross-shaped rosettes in the middle section of the slab, between the lines of the fibre optics. Such a system was applied both to the surface of the concrete under tension and the outer side of the composite. In addition, in the case of a composite strengthened element, 6 strain gauges evenly spaced along the length of the element on the tensometric rosette axes were glued. The exact arrangement of fibre optic and strain gauges used in the described tests is shown in Figure 1. Fibre optic sensors are marked blue, strain gauges applied on concrete and composite surfaces are marked red, while the strain gauges that appear on composite surface only are marked black.

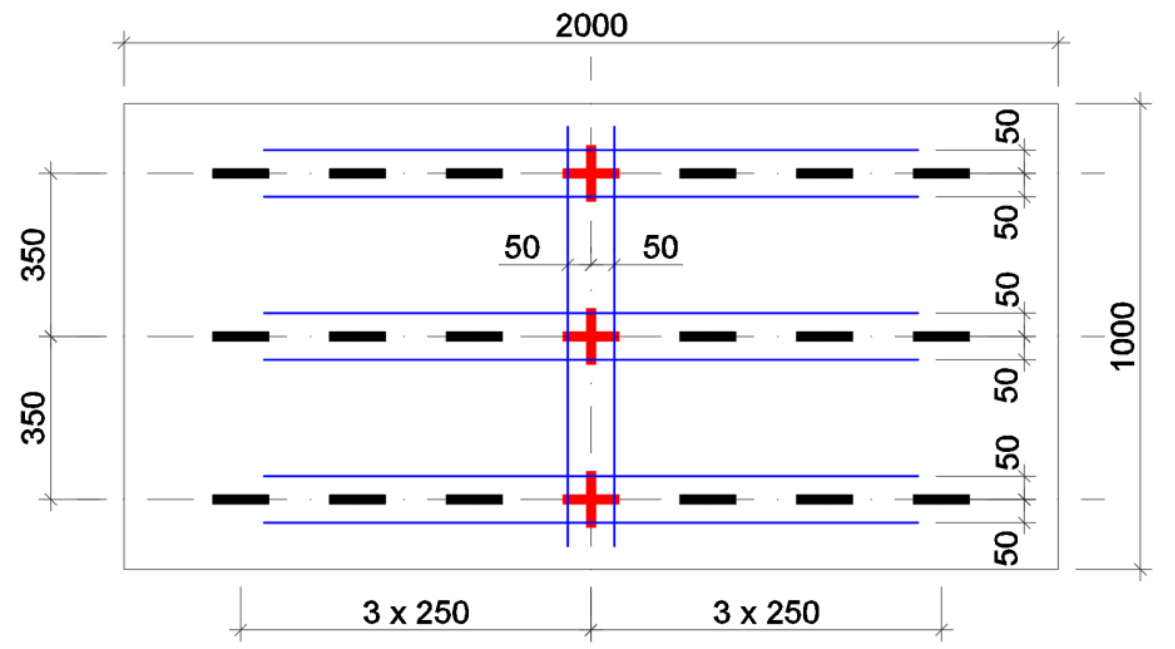

Fig. 1. Diagram of arrangement of measurement sensors used in tests (dimensions in mm).

In addition to the deformation measurements described above, deformations of reinforcing bars, deformations of the compressed concrete surface and displacements of the element in the middle of the span and over the supports were also monitored. However, the results obtained are not related to the content of this publication, and therefore they have not been discussed here.

\subsection{Preparation of elements for testing}

The basic research elements were prepared at the prefabrication plant, where sensors responsible for measuring deformations on steel were also installed. Before the strengthening was applied, the elements were sanded and cleaned to ensure better adhesion of the FRCM composite to the concrete. Before mounting fibre optic and strain gauge sensors, their installation locations were precisely levelled, cleaned and degreased.

Before gluing strain gauges, the surface was primed with the glue provided by the manufacturer. After the glue had dried on the concrete surface, the gluing of the strain gauges to the element was initiated. The strain gauges that were in the strengthening composite joint were covered in wax to protect the paper strain gauge from the negative effects of the mixing water from the mineral mortar used 
in the composite. An additional difficulty when installing strain gauges under the composite layer was the necessity of routing the cables in such a way as to minimize their effect upon the FRCM strengthening work.

The first stage of the installation of fibre optic sensors was their preliminary fixing in accordance with the planned route. The optical fibre was fixed with the use of adhesive tape at characteristic points, and given the appropriate tension to ensure a straight and even measuring line. The optical fibre was glued with a two-component epoxy glue, which was spread evenly using a plastic spatula. After its initial setting, temporary assembly tapes were removed and epoxy glue was added in their places. The stages of preparing fibre optic sensors are shown in Figures 2 and 3.

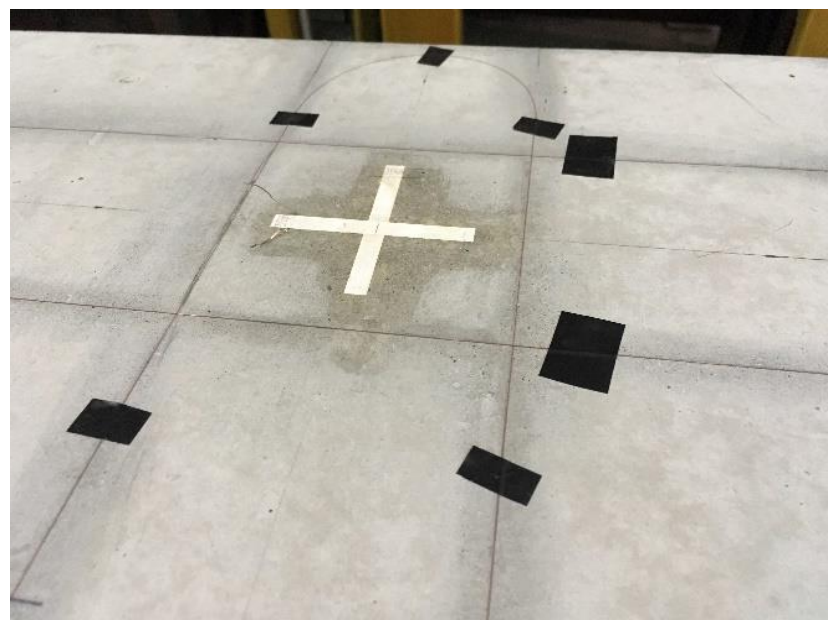

Fig. 2. Fibre optics pre-attached to the concrete surface and strain gauges glued to it.

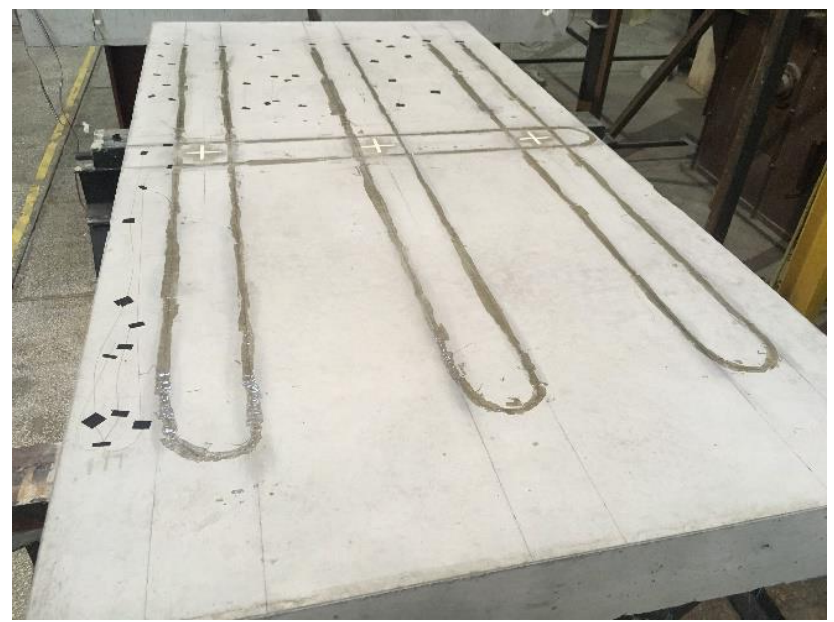

Fig. 3. View of the entire test element with the finally attached fibre optics.

Due to covering the optical fibre with a layer of epoxy glue, it was not necessary to additionally protect it from damage. In the case of fibre optic sensors in the composite joint, the roughness of the epoxy glue surface was increased by means of fine quartz sand spilled onto the glue during its bonding. Because of the absence of connecting wires in the DFOS method, fibre optic ends were put on the side of the element. 


\section{Results and Discussion}

Both test elements were loaded until they were destroyed. The load was continuously increased with stops at every $10 \mathrm{kN}$ in order to read the fibre optic sensors. In the case of the unstrengthened element, the destruction occurred due to the yielding of the main reinforcing steel, and in the case of the element strengthened with the FRCM composite, it was related to the slippage of the composite after the destruction of its anchorage.

\subsection{Concrete surface cracking}

On the basis of measurements of longitudinal deformation of the concrete under tension, in the middle of the element, the moment of cracking and the manner of its development were observed. The place of the cracking formation in optical fibre measurements is visible in the form of a large local peak that can be seen on the graph. Figure 4 presents three consecutive measuring cycles at the moment of cracking in the element not strengthened with the FRCM composite. The black colour indicates the deformation of the element before cracking, the green colour indicates the first measurement with noticeable cracking. The next stage in the cracking development is marked in red. Main cracking locations are marked with a blue dotted line.

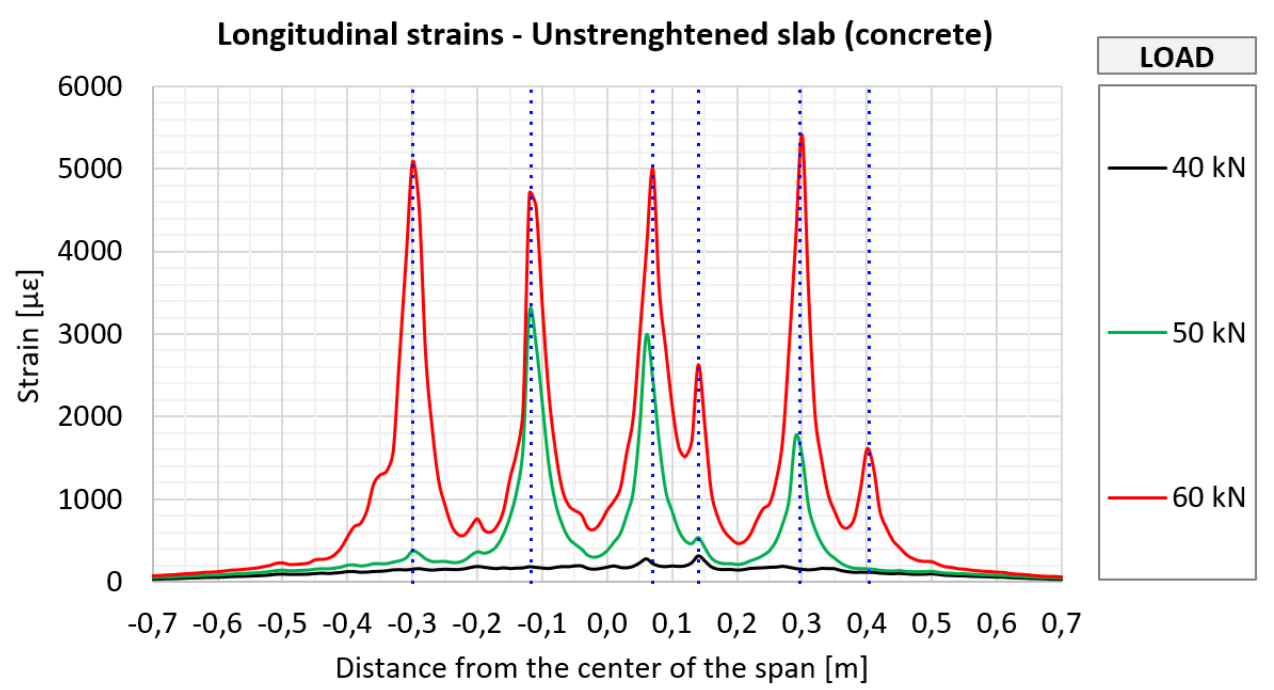

Fig. 4. Distribution of longitudinal deformation of the concrete under tension for a non-strengthened element before and after cracking.

The non-strengthened element was cracked between values of $40 \mathrm{kN}$ and $50 \mathrm{kN}$ of the loading force. Initially, 3 main cracks were observed, the width of which developed at similar speed. After a slight increase in loads, the fourth main crack and two smaller cracks appeared. A similar measurement was made for the element strengthened with the FRCM composite, where the deformation of the concrete underneath the strengthening layer was determined. Its results are shown in Figure 5, with the markings according to the graph in Figure 4. 


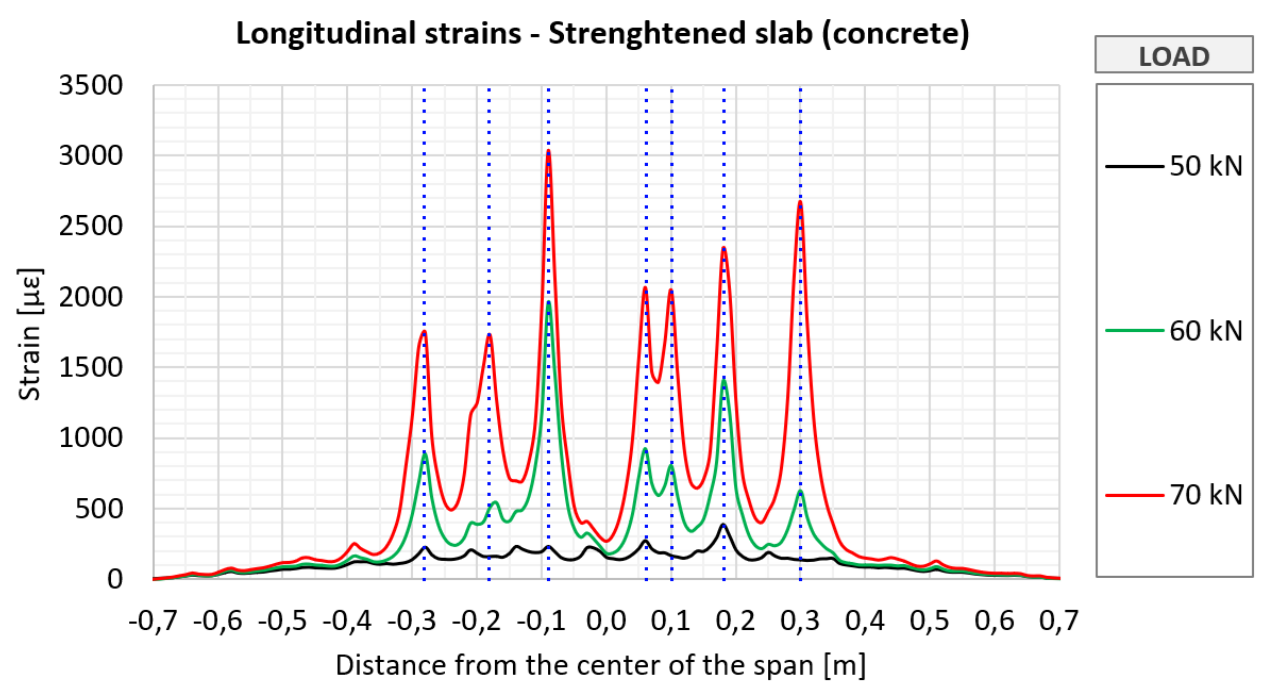

Fig. 5. Distribution of longitudinal deformation of the concrete under tension for a strengthened element before and after cracking.

Cracking of the strengthened element occurred later than in the case of an element without strengthening, and this took place between a load of $50 \mathrm{kN}$ and $60 \mathrm{kN}$. It also had a different character than the non-strengthened element cracking, as in the first cracking phase more cracks appeared. During further loading of the element, 7 main cracks with a similar level of deformation could be seen, which indicates that the elements strengthened with FRCM composite are marked by a more favourable, denser distribution of cracks with a smaller width.

The character and the moment of cracking measured in both cases were consistent with the visual evaluation carried out during the tests. With a more detailed analysis of the graphs depicted in Figures 4 and 5, it can be seen that the possible locations of future cracks are already visible before actual cracking of the concrete surface occurs. Such observations can be helpful in predicting cracks as part of the structural health monitoring. DFOS readings also allow you to observe cracks in invisible elements. The surface of the concrete covered in the FRCM composite was cracked, which was analysed without removing the actual strengthening.

\subsection{Deformation of FRCM composite}

An important aspect of FRCM strengthening composites work is their deformation. In fibre optic measurement, three separate stages of deformation development were observed. In the first phase, when the concrete is not cracked, the composite also works in a noncracked state. The second phase of the composite work begins after the reinforced concrete element becomes cracked. There is a visible increase in the deformation of the composite in places where the mineral matrix yields to microcracks. The next phase of deformation is visible after full cracking of the composite. Further increase of loads results in proportional increase in deformations, until detachment or slip of the composite, which lead to the destruction of the element. The described deformation development of the FRCM composite is illustrated in Figure 6. The shades of green are used to mark phase 1 , before the occurrence of cracks, phase 2 is marked by shades of blue, and phase 3 by shades of red. 


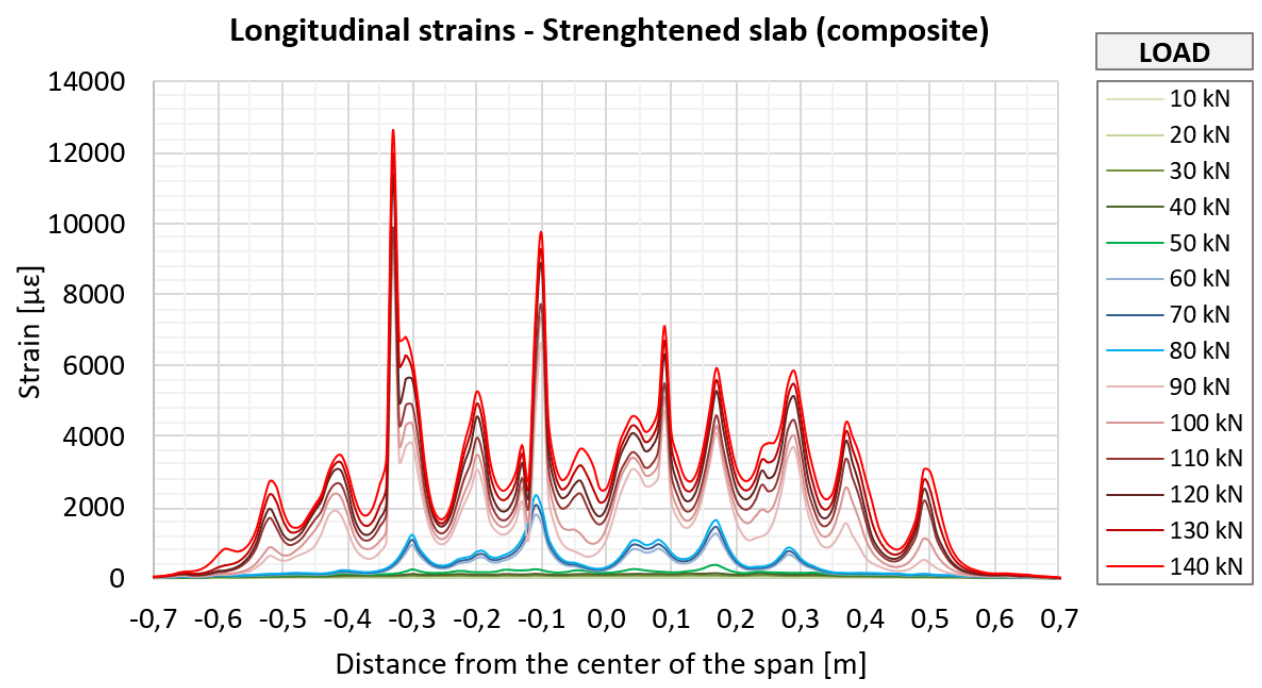

Fig. 6. Distribution of longitudinal deformation of the composite.

\subsection{Comparison of deformation measurements of the composite made by means of the DFOS and strain gauges}

The analysis of the work of the composite described in the previous subsection would not have been possible with the use of point measurements made with strain gauges. Interpolation of the results does not allow you to observe the development of cracks, and placing the sensor in the wrong place may result in unreliable measurements. Figure 7 presents a comparison of deformation measurements of the composite made by both fibre optics and strain gauges. The results presented include the range of loads till the first cracking of the element.

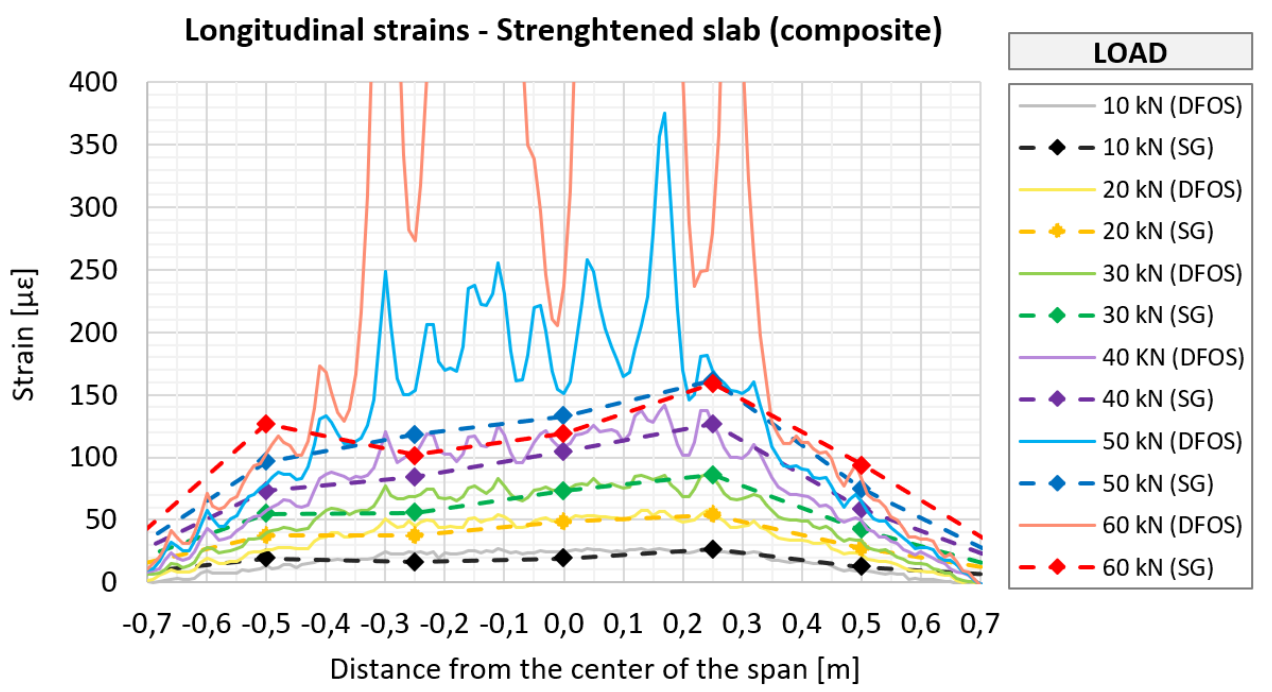

Fig. 7. Comparison of deformation measurement of the composite made with the use of distributed fibre optic sensors (DFOS) and strain gauges (SG).

Deformation values determined by means of fibre optics and strain gauges almost perfectly match until the load of $40 \mathrm{kN}$ is applied. At higher load values, when the first microcracks 
begin to appear, the readings from strain gauges miss some of the significant deformation values. The line corresponding to the load value of $50 \mathrm{kN}$ and $60 \mathrm{kN}$ matches almost exactly the actual deformation distribution only in places where there is no cracking of the surface. As the load on the element was further increased, the observed differences were significantly greater. This is due to the random location of cracks, which may occur in the scope of the strain gauge measuring base or outside it.

\section{Conclusions}

The research involved testing the usefulness of the DFOS method in determining the effectiveness of FRCM composite strengthening. Based on them, the following conclusions were drawn:

- A great advantage of DFOS measurements is their geometric continuity, which allows for measuring deformations of the composite in many places. This is especially important in the case of strengthening composites, which are subject to greatest deformations at the places of cracks, which cannot be accurately determined before the test,

- An additional benefit of using optical fibre measurements is the ability to accurately determine the place of cracking the element based on the peaks visible in the diagrams. With a sufficiently dense distribution of sensors, it is possible to determine the exact morphology of the cracking in the tested element,

- Absence of additional wiring of fibre optic sensors causes less disturbance of the element's surface if a sensor is placed in the composite joint,

- The main disadvantage of DFOS measurements is the need to temporarily suspend the test in order to read the results. Continuous measurement is impossible; such measurement can be performed with strain gauges,

- With larger crack widths, a large influence of the local extraction of the optical fibre from the glue is visible, which causes a strong reading disruption when the element is destroyed. However, it should be noted that in the case of even a small crack, strain gauges are most often damaged,

- Sensitivity of optical fibres to mechanical damage makes it difficult to use them inside the element due to the risk of damage when concreting the element. Strain gauges can be easily protected, and in the case of optical fibres, it is necessary to place them inside a composite reinforcing bar, which can significantly disturb the measurement results,

- Both described methods of measuring deformation give strongly disturbed results in the case of large cracks in elements, which makes it problematic to analyse the moment of the element's destruction. The exact results can be obtained with small cracks corresponding to the actual designed work of the element.

DFOS tests have both advantages and disadvantages compared to strain gauges. Both methods can be used to read essential work parameters, which are useful while analysing the effectiveness of FRCM strengthening. Simultaneous and well-thought-out application of both methods allows for more complete results for later analysis.

The work was created as part of the research project PWr-RUDN 2017, carried out by the Wrocł aw University of Science and Technology together with the Russian University of Friendship of Nations in Moscow. 


\section{References}

1. T. Trapko, Composites Part B, 54, 138-145 (2013)

2. O. Awani, T. El-Maaddawy, N. Ismail, Constr. Build. Mater., 132, 94-111 (2017)

3. F. G. Carozzi, C. Poggi, Composites Part B, 70, 215-230 (2015)

4. T. D'Antino, L. H. Sneed, C. Carloni, C. Pellegrino, Constr. Build. Mater., 101, 838$850(2015)$

5. T. Trapko, M. Musiał, Composites Part B, 118, 67-74 (2017)

6. D. Marcinczak, T. Trapko, M. Musiał, Composites Part B, 158, 149-161 (2019)

7. A. Rogers, Meas. Sci. Technol., 10, R75-R99 (1999)

8. S. Delepine-Lesoille, E. Merliot, C. Boulay, L. Quétel, M. Delaveau, A. Courteville, Smart Mater. Struct., 15, 931-938 (2006)

9. J. M. López-Higuera, L. R Cobo, A. Q. Incera, A. Cobo, J. Light. Technol., 29, 587$608(2011)$

10. C. K. Y. Leung, NDT and E Int., 34, 85-94 (2001)

11. W. Li, X. Bao, High spatial resolution distributed fiber optic technique for strain and temperature measurements in concrete structures, International Workshop on Smart Materials \& Structures, SHM and NDT for the Energy Industry (Calgary, Canada 2013)

12. Ruredil X Mesh Gold Data Sheet, Ruredil SPA (Milan, Italy 2009) 